\title{
DETERMINAÇÃO DE MISTURAS DE SULFAMETOXAZOL E TRIMETOPRIMA POR ESPECTROSCOPIA ELETRÔNICA MULTIVARIADA
}

\author{
Gilcélia A. Cordeiro e Patricio Peralta-Zamora* \\ Departamento de Química, Universidade Federal do Paraná, CP 19081, 81531-990 Curitiba - PR, Brasil \\ Noemi Nagata \\ Departamento de Química, Universidade Estadual de Ponta Grossa, Ponta Grossa - PR, Brasil \\ Roberto Pontarollo \\ Departamento de Farmácia, Universidade Federal do Paraná, Curitiba - PR, Brasil
}

Recebido em 21/12/06; aceito em 2/8/07; publicado na web em 19/12/07

\begin{abstract}
DETERMINATION OF SULFAMETHOXAZOLE AND TRIMETHOPRIM MIXTURES BY MULTIVARIATE ELECTRONIC
SPECTROSCOPY. In this work a multivariate spectroscopic methodology is proposed for quantitative determination of sulfamethoxazole and trimethoprim in pharmaceutical associations. The multivariate model was developed by partial least-squares regression, using twenty synthetic mixtures and the spectral region between 190 and $350 \mathrm{~nm}$. In the validation stage, which involved the analysis of five synthetic mixtures, prediction errors lower that $3 \%$ were observed. The predictive capacity of the multivariate models is seriously affected by spectral changes induced by $\mathrm{pH}$ variations, a fact that acquires a great significance in the analysis of real samples (pharmaceuticals) that contain chemical additives.
\end{abstract}

Keywords: sulfamethoxazole-trimethoprim; multivariate calibration; electronic spectroscopy.

\section{INTRODUÇÃO}

A Química Analítica enfrenta com bastante freqüência o desafio proveniente das mais variadas áreas da ciência, sempre com o objetivo de contribuir com o estabelecimento de metodologias analíticas cada vez mais sensíveis, seletivas, confiáveis e de menor custo.

Por motivos óbvios, o controle de qualidade de produtos farmacêuticos adquire grande importância, não apenas para avalizar os processos de produção, mas principalmente para assegurar o cumprimento de padrões qualitativos e quantitativos que garantam a eficácia e segurança dos medicamentos. Deste ponto de vista, a disponibilização de metodologias analíticas confiáveis e, se possível, rápidas e de baixo custo, mostra-se extremamente importante, particularmente no Brasil, que ocupa a décima colocação no ranking dos maiores consumidores de medicamentos no mundo ${ }^{1}$.

$\mathrm{Na}$ área de produção de medicamentos, a associação de dois ou mais fármacos é uma prática bastante freqüente, uma vez que pode otimizar o tratamento e melhorar a adesão do paciente à terapia prescrita. Nestes casos, a quantificação de uma espécie na presença de excipientes e princípios auxiliares pode ser bastante complexa, o que geralmente obriga a utilização de técnicas cromatográficas $^{2}$, reconhecidamente demoradas e dispendiosas.

A espectroscopia eletrônica (UV-Vis) apresenta um conjunto de características que favorecem sua participação em rotinas de análise química. Dentre outras, é possível destacar sua simplicidade operacional, elevada velocidade analítica, baixo custo e possibilidades de uso em sistemas de controle on-line ${ }^{3,4}$. Infelizmente, os frequientes problemas de interferência espectral observados na espectroscopia UV-Vis representam limitações intransponíveis para as metodologias convencionais de calibração, o que inviabiliza a consolidação da técnica como ferramenta de controle.

Nos últimos anos, a calibração multivariada tem sido utilizada com sucesso no estabelecimento de metodologias analíticas fun-

*e-mail: zamora@quimica.ufpr.br damentadas em espectroscopia UV-Vis ${ }^{5-7}$. De maneira geral, observa-se que aproximações multivariadas permitem explorar toda a informação fornecida pela técnica instrumental, o que favorece o desenvolvimento de modelos de calibração confiáveis, mesmo em situações de extrema sobreposição espectral ou complexidade de sinal.

Embora exemplos de aplicação sejam abundantes, observa-se que na área de controle de qualidade de produtos farmacêuticos as aplicações da análise multivariada são bastante reduzidas, principalmente em razão do pequeno número de metodologias validadas. Para melhor avaliação da potencialidade dos processos de calibração multivariada na análise de produtos farmacêuticos, recomenda-se o trabalho de Forina et al. ${ }^{8}$, publicado em 1998.

Em função destes antecedentes, o presente trabalho propôs o desenvolvimento de metodologias analíticas orientadas ao controle de qualidade de produtos farmacêuticos, associando a técnica de espectroscopia eletrônica (UV-Vis) com processos de calibração multivariada (Regressão de Mínimos Quadrados Parciais, PLSR). Como objeto de estudo, foi selecionada a associação Sulfametozaxol (SMZ) e Trimetoprima (TMP), importantes fármacos utilizados no tratamento de infecções do trato respiratório superior e inferior (ex. bronquite, faringite, sinusite e otite), do trato urinário e do sistema gastrointestinal ${ }^{9}$.

No Brasil, as normas para a análise desta associação são estabelecidas pela Agência Nacional de Vigilância Sanitária (ANVISA), as quais são baseadas na Farmacopéia Brasileira. De maneira geral, as metodologias sugeridas para identificação e quantificação de fármacos correspondem a técnicas espectroscópicas e cromatográficas, respectivamente ${ }^{10}$.

Dentre as novas metodologias propostas para análise desta associação, destacam-se técnicas fundamentadas em cromatografia líquida $^{11,12}$ e eletroforese capilar ${ }^{13}$. Recentemente, uma metodologia espectroscópica multivariada foi proposta para a quantificação de misturas de sulfanilamidas por espectroscopia UV-Vis, metodologia que permitiu a obtenção de erros de previsão inferiores a $11 \%{ }^{14}$. 


\section{PARTE EXPERIMENTAL}

\section{Materiais e reagentes}

Os fármacos utilizados neste estudo foram Sulfametoxazol (SMZ: 4-amino-N-(5-metil-3-isoxazolil) benzenossulfonamida) e Trimetoprima (TMP: 5-[(3,4,5-trimetoxifenil)metil]-2,4pirimidinodiamina), ambos fornecidos pelo laboratório farmacêutico Far-Manguinhos da Fundação Oswaldo Cruz, com grau de pureza de $99 \%$. A estrutura química destes fármacos é apresentada na Figura 1.

Etanol (98,9\%, Vetec), acetronitrila (grau HPLC, Biotec), trietilamina (grau HPLC, Biotec) e metanol (grau HPLC, J.T. Baker) foram utilizados como recebidos.

Outros reagentes (ácidos, bases e solventes) foram de grau analítico PA.

A água deionizada foi de qualidade Milli- $\mathrm{Q}^{\circledR}$.

Todas as soluções foram preparadas utilizando-se vidraria analítica, incluindo balões, buretas e pipetas volumétricas previamente calibradas.

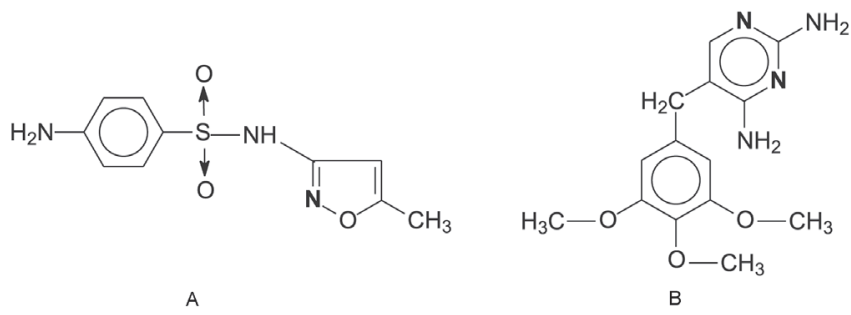

Figura 1.Estrutura química do sulfametoxazol (A) e da trimetoprima (B).

\section{Equipamentos}

Espectros de absorção UV-Vis foram obtidos em espectrofotômetro Shimadzu (2410 PC), utilizando-se o software UVPC v.3.91 (Shimadzu) e cubetas de quartzo de $1 \mathrm{~cm}$.

A análise por cromatografia líquida de alta eficiência foi realizada em um cromatógrafo Varian Pró-Star, equipado com coluna C18 (Chrompack) e detector UV (213 nm). Como fase móvel foi utilizada uma mistura de acetonitrila $(222 \mathrm{~mL})$, água deionizada (777 mL) e trietilamina $(1,1 \mathrm{~mL})$, com vazão de $1 \mathrm{~mL} \mathrm{~min}^{-1}$. Curvas de calibração individuais foram preparadas a partir de padrões puros (USP).

Todas as pesagens foram realizadas em balança analítica digital Scientech AS $210( \pm 0,0001 \mathrm{~g})$.

\section{Programas computacionais}

Para a montagem de matrizes de dados, utilizou-se o software Origin Pro $6.1^{\circledast}$, enquanto que para a elaboração dos modelos empregou-se o programa PLS-toolbox 3.0, que opera em ambiente Matlab v.6.5.

\section{Desenvolvimento dos modelos de calibração}

Para o desenvolvimento de modelos univariados convencionais foram elaboradas curvas analíticas individuais, no comprimento de onda de máxima absorção apresentado por cada substrato. Cada curva de calibração foi composta por 6 valores de concentração, cobrindo-se a faixa de 10,0 a $27,0 \mathrm{mg} \mathrm{L}^{-1}$ para sulfametoxazol, e de 2,0 a $5,0 \mathrm{mg} \mathrm{L}^{-1}$ para trimetoprima. As soluções foram preparadas por dissolução direta dos substratos, em solução etanol:água $5 \%$ (v/v).
Modelos univariados foram também elaborados no modo derivativo. Neste caso, curvas de calibração para sulfametoxazol foram elaboradas nos comprimentos de onda em que trimetoptima apresenta uma derivada da absorvância igual a zero. $\mathrm{O}$ mesmo procedimento foi utilizado para a elaboração das curvas de calibração de trimetoprima.

Para o desenvolvimento de modelos de calibração multivariada foram produzidas 25 misturas, contendo 18 a $22 \mathrm{mg} \mathrm{L}^{-1} \mathrm{de}$ sulfametoxazol e 3 a $5 \mathrm{mg} \mathrm{L}^{-1}$ de trimetoprima, dissolvidas em solução etanol:água $5 \%(\mathrm{v} / \mathrm{v})$, conforme o planejamento de experimentos apresentado na Tabela 1. Esta faixa de trabalho foi definida levando-se em consideração o teor nominal dos fármacos presentes nos medicamentos comercialmente disponíveis, assim como a variação máxima permitida pela legislação pertinente $( \pm 10 \%)$. O modelo multivariado foi desenvolvido utilizando-se 20 misturas, sendo que as 5 misturas restantes, selecionadas aleatoriamente, foram reservadas para a fase de validação.

Os espectros foram registrados entre 190 e $400 \mathrm{~nm}$, utilizandose cubetas de quartzo de $1 \mathrm{~cm}$ de caminho ótico, sendo processados integralmente, eliminando-se, apenas, regiões sem informação relevante. Para elaboração de todos os modelos multivariados, os dados espectrais foram centrados na média (translação do sistema de origem até o centro do conjunto de dados), visando facilitar a visualização, bem como reduzir a dimensão do modelo construído. Este tipo de pré-processamento consiste, basicamente, na subtração do valor de cada elemento da coluna $\left(\mathrm{x}_{\mathrm{ij}}\right)$ pelo valor médio dos elementos dessa coluna $\left(\mathrm{xm}_{\mathrm{j}}\right)$, obtendo-se como resultado uma matriz onde toda a coluna tem média zero ${ }^{15}$.

Tabela 1. Composição das misturas utilizadas nas etapas de calibração e validação (negrito), no desenvolvimento de modelos multivariados, utilizando-se espectroscopia UV-Vis em solução

\begin{tabular}{|c|c|c|}
\hline Misturas & $\operatorname{SMZ}\left(\mathrm{mg} \mathrm{L}^{-1}\right)$ & $\mathrm{TMP}\left(\mathrm{mg} \mathrm{L}^{-1}\right)$ \\
\hline 1 & 18,0 & 3,0 \\
\hline 2 & 19,0 & 3,0 \\
\hline 3 & 20,0 & 3,0 \\
\hline 4 & 21,0 & 3,0 \\
\hline 5 & 22,0 & 3,0 \\
\hline 6 & 18,0 & 3,4 \\
\hline 7 & 19,0 & 3,4 \\
\hline 8 & 20,0 & 3,4 \\
\hline 9 & 21,0 & 3,4 \\
\hline 10 & 22,0 & 3,4 \\
\hline 11 & 18,0 & 4,0 \\
\hline 12 & 19,0 & 4,0 \\
\hline 13 & 20,0 & 4,0 \\
\hline 14 & 21,0 & 4,0 \\
\hline 15 & 22,0 & 4,0 \\
\hline 16 & 18,0 & 4,4 \\
\hline 17 & 19,0 & 4,4 \\
\hline 18 & 20,0 & 4,4 \\
\hline 19 & 21,0 & 4,4 \\
\hline 20 & 22,0 & 4,4 \\
\hline 21 & 18,0 & 5,0 \\
\hline 22 & 19,0 & 5,0 \\
\hline 23 & 20,0 & 5,0 \\
\hline 24 & 21,0 & 5,0 \\
\hline 25 & 22,0 & 5,0 \\
\hline
\end{tabular}




\section{Análise de amostras reais}

Para avaliação dos modelos desenvolvidos, amostras de medicamentos foram analisadas. Além do medicamento de referência (Bactrim do Laboratório Roché), foram analisadas amostras de BacSulftrim, ambas adquiridas no comércio local. Todas as amostras contêm 400 mg de SMZ e 80 mg de TMP por comprimido.

$\mathrm{Na}$ análise por espectroscopia UV-Vis, os comprimidos foram triturados em almofariz e dissolvidos em 1000,0 mL de solução aquosa de etanol $(5 \% \mathrm{v} / \mathrm{v})$. Após filtração, $5,0 \mathrm{~mL}$ desta solução foram diluídos até $100 \mathrm{~mL}$, utilizando-se a mesma solução aquosa de etanol. Todos os ensaios foram realizados em triplicata.

Para análise cromatográfica, 10 comprimidos de cada medicamento foram triturados, pesando-se o equivalente a $80 \mathrm{mg}$ de SMZ e diluindo-se em $50 \mathrm{~mL}$ de metanol, previamente sonicado por 5 min. Após filtração em membrana de $45 \mu \mathrm{m}, 1 \mathrm{~mL}$ desta solução foi transferida para um balão de $10 \mathrm{~mL}$, diluindo-se com fase móvel.

\section{Validação do modelo multivariado}

A partir da análise comparativa dos modelos de calibração desenvolvidos com misturas dos padrões de SMZ e TMP, o melhor modelo foi selecionado, sendo validado segundo critérios estabelecidos pela ANVISA. Os indicadores foram precisão (repetibilidade e reprodutibilidade), recuperação e robustez (tempo de leitura, temperatura e influência do $\mathrm{pH}$ ).

\section{RESULTADOS E DISCUSSÃO}

\section{Calibração convencional}

A séria interferência espectral existente entre ambas espécies em estudo pode ser observada na Figura 2, que apresenta os espectros eletrônicos das substâncias puras, em concentrações que mantêm a proporção de dosagem dos medicamentos comercializados. SMZ apresenta um máximo em 265,5 nm, praticamente livre de interferências por parte do TMP. Por sua vez, TMP apresenta um máximo com severa interferência em $203,5 \mathrm{~nm}$ e um ombro de interferência mínima, em 236,0 nm.

Em função deste grau de interferência, curvas analíticas individuas foram elaboradas em 265,5 $\mathrm{nm}$ para SMZ $(\mathrm{A}=-0,005+$ $\left.0,072 \times \mathrm{C}_{\mathrm{SMZ}}, \mathrm{R}=0,999\right)$ e $236,0 \mathrm{~nm}$ para TMP $(\mathrm{A}=-0,002+0,050$

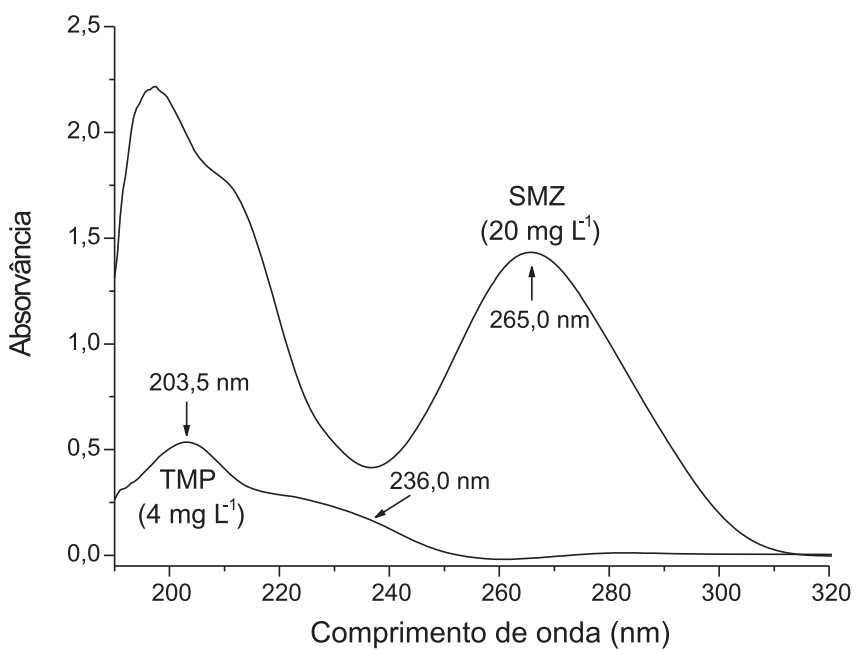

Figura 2. Espectro eletrônico de sulfametoxazol e trimetoprima $\mathrm{x} \mathrm{C}_{\mathrm{TMP}}, \mathrm{R}=0,973$ ), a partir das quais foram obtidos os resultados apresentados na Tabela 2. Como era de se esperar, os erros são extremamente elevados, principalmente para o componente que se apresenta em menor concentração (TMP). Neste caso, a forte interferência espectral do SMZ provoca erros positivos, cuja magnitude demonstra a inviabilidade deste procedimento univariado convencional.

Visando contornar os problemas de interferência espectral, os espectros de absorção UV-Vis foram processados em primeira derivada. Em princípio, curvas de calibração para SMZ podem ser construídas no ponto de derivada zero do TMP $(203,5 \mathrm{~nm}$; A = $\left.-0,0065+0,024 \times \mathrm{C}_{\mathrm{SMZ}}, \mathrm{R}=0,981\right)$, assim como curvas para TMP podem ser elaboradas no ponto de derivada zero do SMZ (236,5 $\left.\mathrm{nm} ; \mathrm{A}=-0,003+0,0017 \times \mathrm{C}_{\mathrm{TMP}}, \mathrm{R}=0,923\right)$, com um mínimo de interferência. Infelizmente, valendo-se deste artifício, denominado método do ponto de anulação, os sinais processados apresentam magnitudes bastante reduzidas, o que diminui significativamente a linearidade das curvas analíticas. Os resultados obtidos na análise de misturas sintéticas são apresentados na Tabela 2. Embora o procedimento adotado permita reduzir significativamente os erros originados pela interferência espectral, observa-se uma correção incompleta, principalmente em razão da perda de linearidade observada no processamento de sinais de baixa intensidade.

Finalmente, para complementar os procedimentos ditos "convencionais" e contornar os já comentados problemas de interferência espectral, sistemas fundamentados no princípio da aditividade das absorvâncias foram também desenvolvidos, de acordo com a seqüência de expressões apresentadas a seguir:

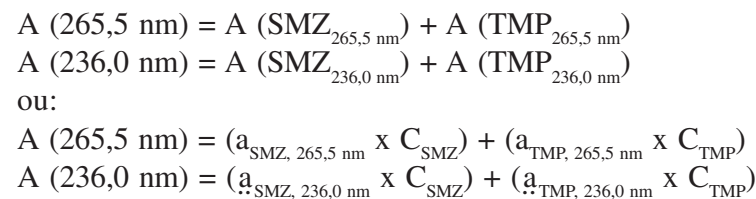

onde: A - Absorvância, ạ - absortividade $\left(\mathrm{L} \mathrm{mg}^{-1} \mathrm{~cm}^{-1}\right), \mathrm{C}$ - concentração $\left(\mathrm{mg} \mathrm{L}^{-1}\right)$

Registrando-se os valores de absorvância para a mistura de substratos, em ambos comprimentos de onda, e conhecendo-se os valores de absortividade, é possível calcular as concentrações, resolvendo-se um sistema de duas equações. Os resultados, apresentados na Tabela 2, demonstram a ineficiência deste procedimento para a correção de interferência espectral tão severa quanto a envolvida neste estudo. Normalmente, a utilização de sistemas de equações fundamentados no princípio da aditividade da absorvância permite contornar problemas de interferência espectral, em sistemas contendo dois ou três componentes. Neste caso, o sistema não viabilizou a determinação desejada, principalmente em razão das grandes diferenças de concentração entre ambas espécies em estudo. Nestas condições, pequenos desvios que possam afetar o sinal do substrato mais concentrado se tornam extremamente significativos, influenciando severamente o sinal correspondente à espécie menos concentrada.

Os elevados erros de previsão observados servem de justificativa para o desenvolvimento de modelos multivariados de calibração, os quais comumente permitem eficiente previsão, mesmo na presença de elevada interferência espectral.

\section{Calibração multivariada}

O modelo multivariado de calibração foi estabelecido a partir de 20 misturas sintéticas, selecionadas aleatoriamente a partir das 25 misturas apresentadas na Tabela 1 . As 5 misturas restantes fo- 
Tabela 2. Resultados das concentrações previstas nas análises de misturas sintéticas contendo SMZ e TMP, por processos de calibração convencional

\begin{tabular}{|c|c|c|c|c|c|c|c|c|}
\hline \multirow[t]{3}{*}{ AMOSTRA } & \multicolumn{2}{|c|}{ Concentração real (mg L'-1) } & \multicolumn{6}{|c|}{ Concentração encontrada $\left(\mathrm{mg} \mathrm{L}^{-1}\right)$} \\
\hline & \multirow[b]{2}{*}{ SMZ } & \multirow[b]{2}{*}{ TMP } & \multicolumn{2}{|c|}{ Método 1} & \multicolumn{2}{|c|}{ Método 2} & \multicolumn{2}{|c|}{ Método 3} \\
\hline & & & SMZ & TMP & SMZ & TMP & SMZ & TMP \\
\hline 2 & 19,0 & 3,0 & 15,9 & 11,4 & 13,6 & 3,5 & 15,7 & 5,1 \\
\hline 9 & 21,0 & 3,4 & 18,1 & 12,8 & 16,1 & 3,4 & 17,8 & 5,7 \\
\hline 17 & 19,0 & 4,4 & 16,8 & 16,4 & 14,9 & 7,5 & 16,3 & 10,6 \\
\hline 20 & 22,0 & 4,4 & 19,1 & 17,5 & 16,9 & 7,2 & 18,6 & 10,7 \\
\hline \multirow[t]{2}{*}{23} & 20,0 & 5,0 & 17,8 & 17,3 & 9,4 & 8,4 & 17,3 & 11,1 \\
\hline & & Erro médio (\%) & 13,2 & 271,9 & $-29,9$ & 44,0 & 15,2 & 108,4 \\
\hline
\end{tabular}

Método 1: interpolação em curvas de calibração individuais; método 2: interpolação em curvas em primeira derivada; método 3: aditividade de absorvâncias.

ram reservadas para a fase de previsão, que visa avaliar a capacidade preditiva dos modelos.

Para definir o melhor número de variáveis latentes (VLs), utilizou-se o método de validação interna cruzada, particularmente a rotina denominada "Leave one out" ${ }^{\text {. }}$. Neste procedimento, um espectro é retirado do conjunto de calibração, sendo utilizado como elemento de previsão. Esta operação é repetida tantas vezes quanto necessário, de maneira a garantir que todos os elementos do conjunto participem como elemento de previsão. Os erros que surgem desta previsão são apresentados como somatória do seu quadrado (PRESS) em função do número de VLs (Figura 3).

Processando-se os espectros sem nenhum tipo de pré-processamento, observa-se que 19 variáveis latentes são necessárias para descrever o modelo que leva aos menores erros de previsão. Entretanto, percebe-se que a partir da segunda VL o erro de previsão é relativamente pequeno (erro médio inferior a 0,9 ), ao mesmo tempo em que as primeiras $10 \mathrm{VLs}$ representam praticamente toda a variância dos dados de concentração. Sendo assim, modelos foram

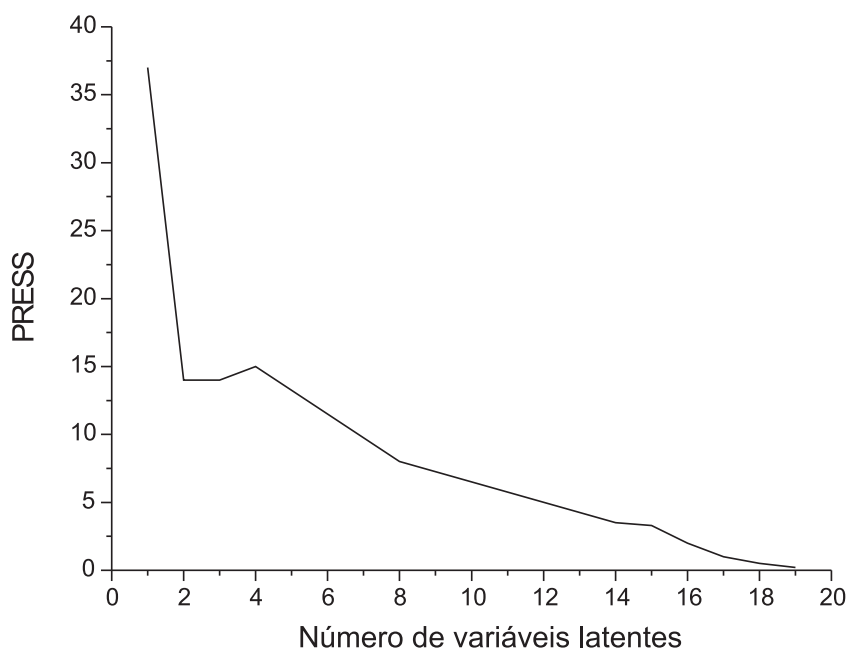

\begin{tabular}{|ccccc|}
\hline \multicolumn{5}{|c|}{ Variância capturada pelo modelo PL\$ } \\
VL & Esta VL & Total $(\%)$ & Esta VL & Total $(\%)$ \\
1 & 92,98 & 92,98 & 33,50 & 33,50 \\
2 & 6,80 & 99,78 & 46,44 & 79,94 \\
3 & 0,14 & 99,92 & 2,16 & 82,10 \\
4 & 0,05 & 99,96 & 5,30 & 87,40 \\
5 & 0,01 & 99,97 & 8,10 & 95,51 \\
6 & 0,01 & 99,98 & 1,61 & 97,12 \\
7 & 0,01 & 99,99 & 1,01 & 98,13 \\
8 & 0,00 & 99,99 & 1,39 & 99,52 \\
9 & 0,00 & 99,99 & 0,22 & 99,74 \\
10 & 0,00 & 99,99 & 0,08 & 99,83 \\
\hline
\end{tabular}

Figura 3. Evolução do PRESS em função do número de variáveis latentes elaborados com 19, 8, 5, 3 e 2 VLs, utilizando-se como critério final de seleção a capacidade de previsão, expressa na forma de erro médio de previsão.

Os resultados (não apresentados) indicaram que, tal como esperado, um número reduzido de VLs leva à elaboração de um modelo com capacidade de previsão similar àquele desenvolvido com um grande número de VLs, o que representa uma vantagem que confere robustez ao modelo desenvolvido. Dentro deste contexto, cabe salientar que o uso de um elevado número de VLs costuma provocar superajustes do modelo, o que faz com que pequenas variações de tipo instrumental possam provocar significativa perda na capacidade de previsão.

É interessante salientar que cada amostra do conjunto é representada por aproximadamente 260 valores de absorvância, registrados em igual número de valores de comprimento de onda. A primeira etapa do procedimento multivariado demonstra que grande parte da variância dos dados pode ser representada por um pequeno número de novas variáveis (VLs), as quais surgem da combinação linear das variáveis originais. Se um pequeno número de VLs é utilizado para representar a variância registrada nos espectros, o desenvolvimento de um modelo de calibração fica extremamente facilitado.

Para melhorar a eficiência dos modelos multivariados envolvendo espectroscopia UV-Vis, alguns procedimentos de transformação de dados foram utilizados. São estes: primeira derivada: permite melhorar a separação de sinais não totalmente sobrepostos; alisamento Savitzky-Golay ${ }^{17}$ : permite a diminuição de ruídos instrumentais; autoescalonamento: processamento que permite evidenciar sinais de menor intensidade.

Os resultados, resumidamente apresentados na Figura 4, demonstram que os menores erros de previsão são viabilizados por modelos multivariados elaborados com 8 VLs e com dados espectrais originais. Nestas condições, erros médios de previsão inferiores a 3\% foram observados para TMP e SMZ, nas amostras reservadas para validação.

Este modelo foi utilizado para previsão de amostras de medicamentos, cujos resultados são apresentados na Tabela 3. Como referência, utilizaram-se os valores declarados na bula do medicamento e os resultados da análise por cromatografia líquida de alta eficiência.

Antes de se analisar estes resultados, é pertinente salientar que as informações contidas na bula são expressas na forma de miligramas de fármaco por comprimido. Entretanto, a massa dos comprimidos é bastante heterogênea, o que implica discrepâncias da concentração quando as análises envolvem dissolução do comprimido. Admitindo-se uma composição homogênea dos comprimidos, as diferenças de massa podem acarretar diferenças de concen- 


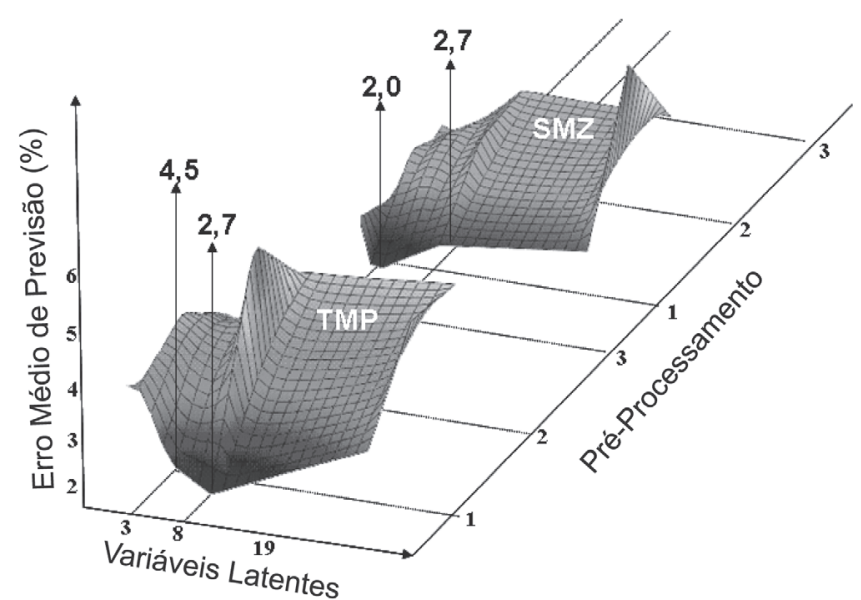

Figura 4. Representação do erro médio de previsão de SMZ e TMP em função do pré-processamento e do número de variáveis latentes

tração da ordem de $2 \%$, em relação ao valor médio.

Os resultados demonstram, em primeiro lugar, que o modelo permite uma excelente previsão do medicamento de referência (Bactrim), com erros inferiores a 7\% em relação aos valores determinados pela técnica cromatográfica padrão. Adicionalmente, observa-se uma elevada reprodutibilidade nos valores da triplicata, para ambas metodologias empregadas, assim como uma boa coerência com os valores declarados na bula do medicamento.

Para o segundo medicamento, o modelo multivariado fornece resultados mais discrepantes, principalmente em razão das significativas diferenças observadas nos respectivos espectros. Em geral, é possível observar uma grande semelhança entre o espectro do medicamento de referência (Bactrim) e a mistura padrão que contém os fármacos em iguais concentrações que as do medicamento. Para o segundo medicamento observa-se um deslocamento da banda centrada em $268 \mathrm{~nm}$, além de uma significativa diminuição de sinal (Figura 5a). Trata-se de uma situação anômala, a qual, em princípio, pode ser atribuída à presença de excipientes.

Estudos posteriores permitiram verificar discretas diferenças de $\mathrm{pH}$ nas amostras solubilizadas dos medicamentos, assim como substanciais modificações do perfil espectroscópico de ambas espécies, quando em diferentes condições de acidez (Figura 5b). Analisando-se a estrutura química dos compostos em estudo, evidenciam-se centros de maior densidade eletrônica, passíveis de protonação. Embora a diferença nos valores de $\mathrm{pH}$ nos diversos medicamentos seja discreta, os deslocamentos provocados pela protonação podem ser relevantes, o que compromete a capacidade de previsão dos modelos desenvolvidos.

Ante tal inconveniente, duas alternativas de minimização podem ser praticadas. A primeira envolve o uso de sistemas tamponados, enquanto que a segunda envolve o desenvolvimento de modelos que incluam os excipientes causadores de referida anomalia.
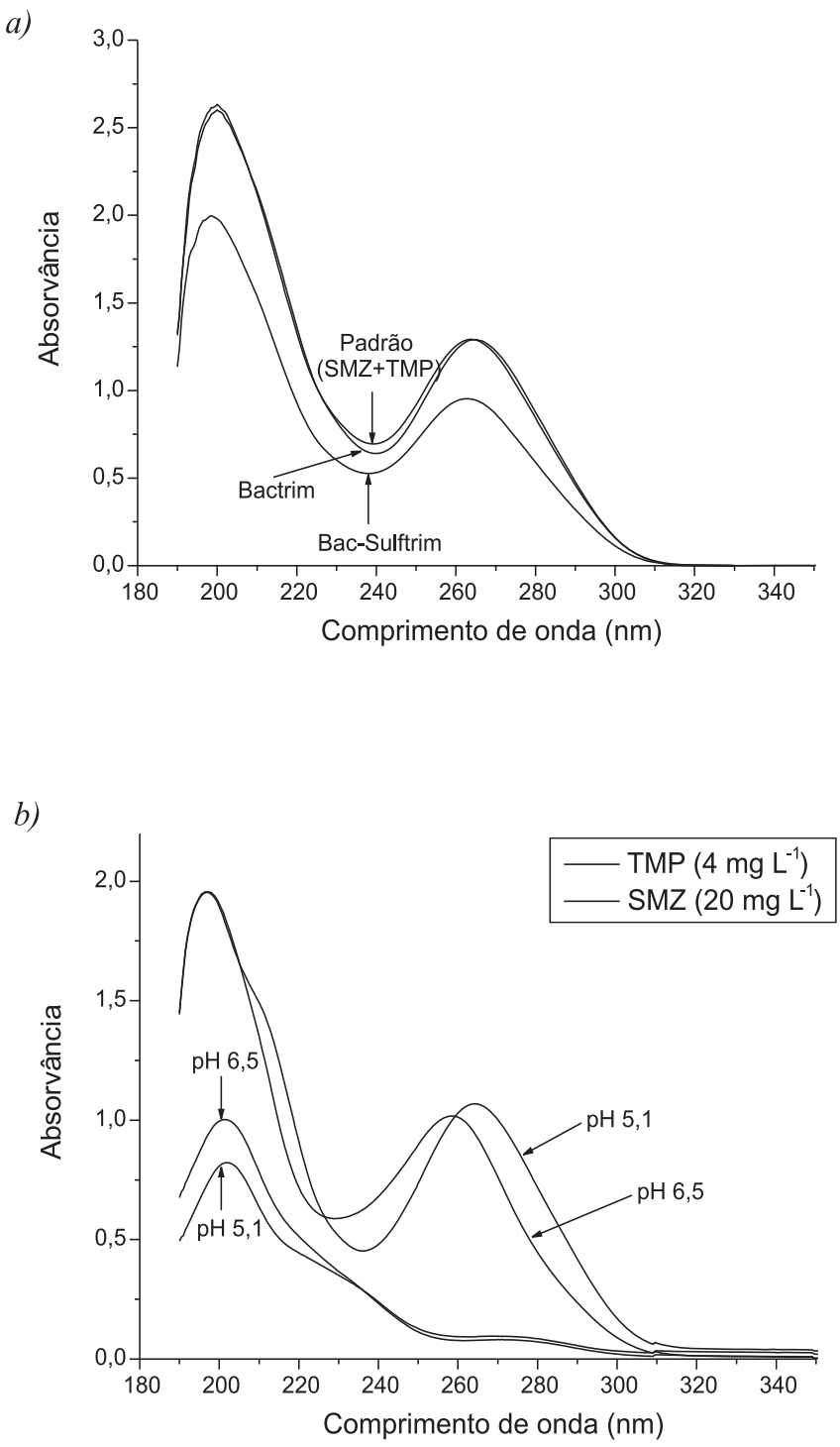

Figura 5.Espectro eletrônico dos medicamentos estudados (a) e efeito do pH no sinal espectral de SMZ e TMP (b)

\section{Estudos de validação}

Visando avaliar as figuras de mérito do modelo multivariado, estudos de validação foram realizados de acordo com critérios es-

Tabela 3. Resultados das concentrações previstas na análise multivariada de medicamentos contendo SMZ e TMP

\begin{tabular}{llccccccc}
\hline Amostras & \multicolumn{2}{c}{$\begin{array}{c}\text { Concentração } \\
\text { Real }\left(\mathrm{mg} \mathrm{g}^{-1}\right)^{1}\end{array}$} & \multicolumn{2}{c}{$\begin{array}{c}\text { Concentração por } \\
\text { CLAE }\left(\mathrm{mg} \mathrm{g}^{-1}\right)^{2}\end{array}$} & \multicolumn{2}{c}{$\begin{array}{c}\text { Concentração } \\
\text { Prevista }\left(\mathrm{mg} \mathrm{g}^{-1}\right)^{3}\end{array}$} & \multicolumn{2}{c}{ Erro $(\%)^{4}$} \\
\cline { 2 - 10 } & SMZ & TMP & SMZ & TMP & SMZ & TMP & SMZ & TMP \\
\hline Bactrim & 789,1 & 157,8 & $839,4(0,5)$ & $151,3(0,3)$ & $777,4(19,5)$ & $156,5(4,0)$ & $-7,4$ & $+3,4$ \\
Bac-Sulftrin & 641,1 & 128,2 & $593,2(1,9)$ & $114,4(1,0)$ & $596,8(13,6)$ & $95,3(3,6)$ & $+0,6$ & $-16,7$ \\
\hline
\end{tabular}

1: Concentração calculada a partir da composição informada na bula do medicamento, considerando-se a massa média dos comprimidos. 2: Concentração determinada por CLAE em análise em triplicata (precisão expressa na forma de estimativa do desvio padrão). 3: Concentração prevista pelo modelo multivariado em análise em triplicata (precisão expressa na forma de estimativa do desvio padrão). 4: Erro relativo em relação ao valor determinado por técnica cromatográfica padrão 
tabelecidos pela ANVISA. De acordo com estas especificações, parâmetros como recuperação, repetibilidade, reprodutibilidade e robustez, frente a mudanças de $\mathrm{pH}$, temperatura e tempo de leitura, foram avaliados. Para realização deste estudo, o melhor modelo multivariado foi selecionado, o qual foi desenvolvido com 8 VL's e a partir de espectros sem pré-tratamento.

\section{Precisão}

$\mathrm{O}$ teste de repetibilidade avalia a diferença entre repetições do mesmo ensaio, executado no mesmo laboratório e pelo mesmo analista. Isto é, nas mesmas condições de amostra, equipamento e analista. Para avaliação deste parâmetro, três misturas sintéticas, contendo concentrações baixas (18,0 $\mathrm{mg} \mathrm{L}^{-1}$ de $\mathrm{SMZ}$ e $3,0 \mathrm{mg} \mathrm{L}^{-1}$ de TMP), médias (20,0 $\mathrm{mg} \mathrm{L}^{-1}$ de SMZ e 4,0 $\mathrm{mg} \mathrm{L}^{-1}$ de TMP) e altas (22,0 $\mathrm{mg} \mathrm{L}^{-1}$ de SMZ e 5,0 $\mathrm{mg} \mathrm{L}^{-1}$ de TMP), foram analisadas em triplicata. Os resultados apresentados na Tabela 4 demonstram desvios padrão relativos (DPR) inferiores a $5 \%$, portanto dentro dos valores aceitos pela legislação vigente ${ }^{18}$.

Adicionalmente, os resultados apresentados na Tabela 4 demonstram uma excelente capacidade de recuperação dos modelos propostos, com valores entre 99 e $101 \%$, portanto dentro dos valores aceitos pela legislação vigente ${ }^{18}$.

O teste de reprodutibilidade é semelhante ao de repetibilidade, com a diferença que, neste caso, a análise é realizada por outro analista, em outro laboratório e com outro equipamento. Os resultados obtidos neste ensaio são apresentados na Tabela 5, demonstrando a obtenção de desvios padrão relativos (DPR) dentro dos

Tabela 4. Resultados da determinação de sulfametoxazol e trimetoprima no ensaio de repetibilidade

\begin{tabular}{|c|c|c|c|c|}
\hline \multirow[t]{2}{*}{ Amostra } & \multicolumn{2}{|c|}{ Sulfametoxazol } & \multicolumn{2}{|c|}{ Trimetoprima } \\
\hline & Real & Previsto & Real & Previsto \\
\hline Mistura 1 & 18,00 & 18,40 & 3,00 & 3,01 \\
\hline Mistura 1d & 18,00 & 17,98 & 3,00 & 2,86 \\
\hline \multirow[t]{4}{*}{ Mistura 1t } & 18,00 & 17,75 & 3,00 & 3,14 \\
\hline & Média & 18,04 & Média & 3,00 \\
\hline & DPR & 1,83 & DPR & 4,67 \\
\hline & $\begin{array}{c}\text { Recuperação } \\
(\%)\end{array}$ & 100,22 & $\begin{array}{c}\text { Recuperação } \\
(\%)\end{array}$ & 100,00 \\
\hline \multirow[t]{2}{*}{ Amostra } & \multicolumn{2}{|c|}{ Sulfametoxazol } & \multicolumn{2}{|c|}{ Trimetoprima } \\
\hline & Real & Previsto & Real & Previsto \\
\hline Mistura 2 & 20,00 & 20,50 & 4,00 & 4,09 \\
\hline Mistura 2d & 20,00 & 19,72 & 4,00 & 4,00 \\
\hline \multirow[t]{4}{*}{ Mistura 2t } & 20,00 & 20,36 & 4,00 & 3,98 \\
\hline & Média & 20,19 & Média & 4,02 \\
\hline & DPR & 2,06 & DPR & 1,46 \\
\hline & $\begin{array}{c}\text { Recuperação } \\
(\%)\end{array}$ & 100,95 & $\begin{array}{c}\text { Recuperação } \\
(\%)\end{array}$ & 100,50 \\
\hline \multirow[t]{2}{*}{ Amostra } & \multicolumn{2}{|c|}{ Sulfametoxazol } & \multicolumn{2}{|c|}{ Trimetoprima } \\
\hline & Real & Previsto & Real & Previsto \\
\hline Mistura 3 & 22,00 & 21,02 & 5,00 & 4,75 \\
\hline Mistura 3d & 22,00 & 21,80 & 5,00 & 5,21 \\
\hline \multirow[t]{4}{*}{ Mistura 3t } & 22,00 & 22,77 & 5,00 & 4,99 \\
\hline & Média & 21,86 & Média & 4,98 \\
\hline & DPR & 4,01 & DPR & 4,61 \\
\hline & $\begin{array}{c}\text { Recuperação } \\
(\%)\end{array}$ & 99,36 & $\begin{array}{c}\text { Recuperação } \\
(\%)\end{array}$ & 99,60 \\
\hline
\end{tabular}

Tabela 5. Resultados da determinação de sulfametoxazol e trimetoprima no ensaio de reprodutibilidade

\begin{tabular}{|c|c|c|}
\hline Amostra 1 & $\begin{array}{l}\text { Sulfametoxazol } \\
\left(18,00 \mathrm{mg} \mathrm{L}^{-1}\right)\end{array}$ & $\begin{array}{l}\text { Trimetoprima } \\
\left(3,00 \mathrm{mg} \mathrm{L}^{-1}\right)\end{array}$ \\
\hline $\begin{array}{l}\text { Determinações } \\
\text { do analista } 1\end{array}$ & $\begin{array}{l}18,40 \\
17,98 \\
17,75\end{array}$ & $\begin{array}{l}3,01 \\
2,86 \\
3,14\end{array}$ \\
\hline $\begin{array}{l}\text { Determinações } \\
\text { do analista } 2\end{array}$ & $\begin{array}{l}18,12 \\
17,87 \\
18,05\end{array}$ & $\begin{array}{l}2,99 \\
3,04 \\
2,94\end{array}$ \\
\hline $\begin{array}{l}\text { Média }\left(\mathrm{mg} \mathrm{L}^{-1}\right) \\
\text { Desvio Padrão } \\
\text { Relativo (\%) }\end{array}$ & $\begin{array}{c}18,03 \\
1,24\end{array}$ & $\begin{array}{l}2,99 \\
3,16\end{array}$ \\
\hline Amostra 2 & $\begin{array}{l}\text { Sulfametoxazol } \\
\left(20,00 \mathrm{mg} \mathrm{L}^{-1}\right)\end{array}$ & $\begin{array}{l}\text { Trimetoprima } \\
\left(4,00 \mathrm{mg} \mathrm{L}^{-1}\right)\end{array}$ \\
\hline $\begin{array}{l}\text { Determinações do } \\
\text { analista } 1\end{array}$ & $\begin{array}{l}20,50 \\
19,72 \\
20,36\end{array}$ & $\begin{array}{l}4,09 \\
4,00 \\
3,98\end{array}$ \\
\hline $\begin{array}{l}\text { Determinações } \\
\text { do analista } 2\end{array}$ & $\begin{array}{l}19,98 \\
19,17 \\
20,47\end{array}$ & $\begin{array}{l}3,99 \\
4,00 \\
3,97\end{array}$ \\
\hline $\begin{array}{l}\text { Média }\left(\mathrm{mg} \mathrm{L}^{-1}\right) \\
\text { Desvio Padrão } \\
\text { Relativo (\%) }\end{array}$ & $\begin{array}{c}20,03 \\
2,61\end{array}$ & $\begin{array}{l}4,01 \\
1,08\end{array}$ \\
\hline Amostra 3 & $\begin{array}{l}\text { Sulfametoxazol } \\
\left(22,00 \mathrm{mg} \mathrm{L}^{-1}\right)\end{array}$ & $\begin{array}{l}\text { Trimetoprima } \\
\left(5,00 \mathrm{mg} \mathrm{L}^{-1}\right)\end{array}$ \\
\hline $\begin{array}{l}\text { Determinações } \\
\text { do analista } 1\end{array}$ & $\begin{array}{l}21,02 \\
21,80 \\
22,77\end{array}$ & $\begin{array}{l}4,75 \\
5,21 \\
4,99\end{array}$ \\
\hline $\begin{array}{l}\text { Determinações } \\
\text { do analista } 2\end{array}$ & $\begin{array}{l}22,53 \\
20,83 \\
20,71\end{array}$ & $\begin{array}{l}4,93 \\
4,77 \\
5,15\end{array}$ \\
\hline $\begin{array}{l}\text { Média (mg L }{ }^{-1} \text { ) } \\
\text { Desvio Padrão } \\
\text { Relativo (\%) }\end{array}$ & $\begin{array}{c}21,61 \\
4,14\end{array}$ & $\begin{array}{l}4,97 \\
3,82\end{array}$ \\
\hline
\end{tabular}

limites impostos pela norma específica $(<5 \%)$.

\section{Robustez}

A sensibilidade do método multivariado frente a variações externas, nas condições experimentais ou das amostras, constitui um fator tão importante quanto as avaliações dos erros de previsão ${ }^{19}$. A análise da robustez determina as condições próprias em que deve ser desenvolvida a calibração e os fatores que devem ser levados em conta durante a construção do modelo. Considerando que a etapa fundamental do modelamento constitui a obtenção dos espectros das espécies de interesse, a principal observação a ser feita é que quaisquer alterações das condições experimentais ou ambientais podem refletir na resposta espectral, isto pode alterar os resultados da calibração e, conseqüentemente, os resultados de previsão, podendo gerar erros significativos de determinação. Neste trabalho, os parâmetros de robustez do método multivariado avalia- 
dos foram temperatura, tempo de leitura e $\mathrm{pH}$.

$\mathrm{O}$ efeito da temperatura foi avaliado entre 5 e $50{ }^{\circ} \mathrm{C}$, utilizandose a mistura que melhor representa a composição dos medicamentos em estudo (SMZ: 20,00 mg L-1, TMP: 4,0 $\mathrm{mg} \mathrm{L}^{-1}$ ). Os espectros (não apresentados) demonstram um efeito significativo da temperatura no perfil de absorção, o que se manifesta como mudanças na intensidade dos sinais registrados. Submetendo-se os espectros ao modelo multivariado, obtiveram-se resultados que confirmam o indesejado efeito da temperatura, quando os valores praticados se afastam do valor em que o modelo foi desenvolvido $\left(25^{\circ} \mathrm{C}\right)$.

$\mathrm{O}$ fato das amostras terem sido solubilizadas em soluções aquosas de etanol sugere que o efeito da temperatura pode ser uma função de perdas diferenciadas de solvente, durante o processo de leitura. Em razão deste comportamento, a necessidade de se desenvolver modelos em condições que possam ser reproduzidas durante a análise é bastante evidente. Caso aconteçam significativas variações de temperatura, o modelo deverá ser atualizado sob essas novas condições.

A estabilidade da absorção das misturas contendo SMZ e TMP foi avaliada em função do tempo, utilizando-se como referência as misturas contendo concentrações baixas $\left(18,00 \mathrm{mg} \mathrm{L}^{-1}\right.$ de $\mathrm{SMZ}$ e 3,00 $\left.\mathrm{mg} \mathrm{L}^{-1} \mathrm{de} \mathrm{TMP}\right)$, médias (20,00 $\mathrm{mg} \mathrm{L}^{-1}$ de SMZ e 4,00 $\mathrm{mg} \mathrm{L}^{-1} \mathrm{de}$ TMP) e altas (22,00 $\mathrm{mg} \mathrm{L}^{-1}$ de SMZ e 5,00 $\mathrm{mg} \mathrm{L}^{-1}$ de TMP). Os espectros foram registrados imediatamente após preparo (tempo 0) e após 24 e $48 \mathrm{~h}$ de estocagem. Neste estudo, pequenas modificações no sinal espectral foram observadas, principalmente na região centrada em 205 nm (resultados não apresentados). Obviamente, estas diferenças provocam inconsistências nos valores previstos pelo modelo muitivariado, principalmente para as soluções que permaneceram estocadas por maior tempo.

Como comentado anteriormente, mudanças de $\mathrm{pH}$ exercem uma significativa influência no perfil espectroscópico das amostras, principalmente em razão dos possíveis equilíbrios de protonação. Observa-se, inclusive, que moderadas diferenças de $\mathrm{pH}$ provocam significativa mudança no padrão de absorção, o que, por definição, compromete de maneira notória a capacidade de previsão dos modelos multivariados.

Uma vez que os modelos foram desenvolvidos no $\mathrm{pH}$ natural das misturas utilizadas como padrão $(\mathrm{pH} \cong 5,5)$, a capacidade de previsão mostra-se adequada apenas em valores próximos deste pH. Em valores diferentes de $\mathrm{pH}$, a previsão leva a resultados que não atendem à legislação vigente, que permite erros não superiores a $5 \%$.

Ante tal situação, o desenvolvimento de modelos em meio tamponado apresenta-se essencial.

\section{CONCLUSÕES}

Os sérios problemas de interferência espectral observados na espectroscopia UV-Vis inviabilizam a quantificação simultânea de sulfametoxazol e trimetoprima, recorrendo-se a modelos de calibração univariados. Os referidos problemas de interferência podem ser minimizados pela utilização de modelos elaborados em primeira derivada ou empregando-se sistemas de equações que levem em consideração a interferência existente. Embora melhorados, os resultados apresentam discrepâncias acima dos limites im- postos pela legislação vigente.

Praticamente toda a informação relevante contida nos espectros pode ser representada por um conjunto de poucas variáveis latentes. Esta redução de dimensionalidade permite a utilização de toda a informação existente, o que favorece o desenvolvimento de modelos multivariados que contornam os problemas de interferência espectral. Modelos de calibração elaborados por rotinas de regressão de mínimos quadrados parciais (PLSR) apresentam boa capacidade preditiva. Durante a fase de validação, realizada a partir da análise de 5 misturas sintéticas, erros de previsão inferiores a $3 \%$ foram observados, para ambos fármacos em estudo.

O pré-processamento de sinais (ex. primeira derivada, primeira derivada seguida de alisamento e autoescalonamento) não induz significativas melhoras nos modelos de calibração desenvolvidos.

A capacidade preditiva dos modelos multivariados é seriamente comprometida pelas mudanças espectrais ocasionadas por variações de pH. Na análise de amostras reais (medicamentos), este fator cobra especial atenção, em razão da presença de excipientes que modificam referido parâmetro.

\section{AGRADECIMENTOS}

Ao apoio financeiro do Conselho Nacional de Desenvolvimento Científico e Tecnológico (CNPQ, Brasil) MCT- CNPq / MSSCTIE-DECIT-DAF - N $54 / 2005$.

\section{REFERÊNCIAS}

1. http://www.progenericos.org.br, acessada em Junho 2006.

2. Escandar, G. M.; Damiani, P. C.; Goicoechea, H. C.; Olivieri, A. C.; Microchem. J. 2006, 82, 29.

3. Johansson, J.; Cauchi, M.; Sundgren, M.; J. Pharm. Biomed. Anal. 2002, 29, 469.

4. Salvati, R.; Longo, A.; Carotenuto, G.; De Nicola, S.; Pepe, G. P.; Nicolais, L.; Barone, A.; Appl. Surf. Sci. 2005, 248, 28.

5. Damiani, P. C.; Moschetti, A. C.; Rovetto, A. J.; Benavente, F.; Olivieri, A. C.; Anal. Chim. Acta 2005, 543, 192.

6. Ni, Y.; Zhang, G.; Kokot, S.; Food Chem. 2005, 89, 465.

7. Sena, M. M.; Chaudhry, Z. F.; Collins, C. H.; Poppi, R. J.; J. Pharm. Biomed. Anal. 2004, 36, 743.

8. Forina, M.; Casolino, M.C.; de la Pezuela Martinez, C.; J. Pharm. Biomed. Anal. 1998, 18, 21.

9. http://www.ccs.ufsc.br/farmacia/TCCGenericos/sulfametoxazol/ farmacologia, acessada em Abril 2006.

10. U.S. Pharmacopeial Convention.; USP - The United States Pharmacopeia, $25^{\text {th }}$ ed., Rockville, MD, 2002.

11. Akay, C.; Özkan, S. A.; J. Pharm. Biomed. Anal. 2002, 30, 1207.

12. Esteve-Romero, J.; Carda-Broch, S.; Gil-Agustí, M.; Capella-Peiró, M. L.; Bose, D.; TrAC, Trends Anal. Chem. 2005, 24, 75.

13. Berzas Nevado, J. J.; Castañeda Peñalvo, G.; Guzmán Bernardo, F. J.; J. Chromatogr., A 2001, 918, 205.

14. Ni, Y.; Qi, Z.; Kokot, S.; Chemom. Intell. Lab. Syst. 2006, 82, 241.

15. Thomas, E. V.; Anal. Chem. 1994, 66, 795.

16. Martens, H.; Naes, T.; Multivariate Calibration, John Wiley \& Sons: Chichester, 1989.

17. Savitzky, A.; Gollay, M. J. E.; Anal. Chem. 1964, 36, 1627.

18. ANVISA; Resolução $n^{\circ} 899$ de 29 de maio de 2003 que determina a publicação do Guia para validação de métodos analíticos e bioanalíticos, Brasília: Diário Oficial da União, 02 fev. 2003.

19. Swierenga, H.; de Weijer, A. P.; van Wijk, R. J.; Buydens, L. M. C.; Chemom. Intell. Lab. Syst. 1999, 49, 1. 